\title{
Two novel algorithms for wavefront reconstruction from pyramid sensor data: \\ Convolution with Linearized Inverse Filter and Pyramid Fourier Transform Reconstructor
}

\author{
Iuliia Shatokhina ${ }^{\mathrm{a}}$, Victoria Hutterer ${ }^{\mathrm{b}}$, and Ronny Ramlau ${ }^{\mathrm{c}}$ \\ ${ }^{a}$ Industrial Mathematics Institute, Johannes Kepler University Linz, 69 Altenbergerstrasse, \\ Linz, A-4040, Austria \\ bIndustrial Mathematics Institute, Johannes Kepler University Linz, 69 Altenbergerstrasse, \\ Linz, A-4040, Austria \\ 'Industrial Mathematics Institute, Johannes Kepler University Linz, 69 Altenbergerstrasse, \\ Linz, A-4040, Austria
}

\begin{abstract}
In this paper we present two novel algorithms for wavefront reconstruction from pyramid-type wavefront sensor data. Assuming a computationally very challenging setting corresponding to the extreme adaptive optics on the European Extremely Large Telescope, we present the results of the performed end-to-end simulations and compare the achieved AO correction quality (in terms of the Long-Exposure Strehl ratio) to other methods like MVM and P-CuReD. Also, we provide a comparison in terms of applicability and computational complexity and closed loop performance, of our novel algorithms to other methods existing for this type of sensor.
\end{abstract}

Keywords: Pyramid wavefront sensor, wavefront reconstruction algorithms

\section{INTRODUCTION}

In order to image the celestial objects with resolution near the diffraction limit, ELTs will rely on a large number of correcting elements, which make fast and accurate wavefront reconstruction very challenging with respect to the related computational load. For instance, in the XAO system designed for the EPICS instrument on the E-ELT, more than 25.000 actuators need to be controlled with the frame rate of about $3 \mathrm{kHz}$. The conventional algorithm, the matrix vector multiplication (MVM), is associated with a serious computational load which makes it hardly feasible for the high order real time AO correction. Therefore, the development of alternative mathematical algorithms, which would be faster than MVM and provide the same quality of reconstruction, is of crucial importance.

In recent years, the authors with colleagues have developed several novel algorithms based on the mathematical analysis of the forward models of the P-WFS. The gained experience is summarized in. ${ }^{1}$ There one can find a comprehensive description of the P-WFS forward models for all modulation scenarios. Also, it contains four developed algorithms, which reach similar or even higher correction quality than the MVM method. As a quality measure the Long-Exposure (LE) Strehl ratio was used, which is evaluated as the average on-axis Strehl ratio for all performed time steps. At the same time, the developed algorithms are all significantly more efficient in terms of speed.

In this paper we present two novel algorithms for wavefront reconstruction from pyramid-type WFS data. Our methods are flexible and rather general in the sense that they are applicable to both pyramid and roof type sensors, used both with and without modulation. The paper is organized as follows. In Section 2 we present

Further author information: (Send correspondence to Iuliia Shatokhina)

Iuliia Shatokhina: E-mail: iuliia.shatokhina@indmath.uni-linz.ac.at, Telephone: +43 (0)732 24684111

Ronny Ramlau: E-mail: ronny.ramlau@jku.at, Telephone: +43 (0)732 24684101 
an overview of the mathematical forward models of the pyramid sensor data and their approximations. The Fourier domain representation of the considered linearized approximate forward models, derived in Section 3 , bring the reader to Section 4, where two novel algorithms are presented. The closed loop performance of our algorithms is compared to the MVM and the P-CuReD results in Section 5. The computational complexity of our methods is analyzed in Section 6. There we also compare our algorithms to other existing methods for P-WFS data. We compare their closed loop performance and computational complexities, analyze their advantages and weaknesses.

\section{PYRAMID/ROOF WFS FORWARD MODELS}

The full Fourier optics based forward model of the non-modulated P-WFS was first derived in. ${ }^{2}$ According to the theoretical model, the sensor measures a combination of one-dimensional and two-dimensional Hilbert transforms of the non-linear functions of the phase. For the modulated pyramid sensor, the full theoretical model becomes even more complicated. A comprehensive description of the full as well as approximate forward models of pyramid and roof sensors for all modulation scenarios can be found in. ${ }^{1}$ Clearly, such forward models are mathematically difficult to invert. However, several assumptions allow us to simplify the forward model significantly. In the remainder of the section we will focus on these assumptions and the corresponding approximate models which led us to the development of the presented algorithms. We use the notations introduced in. ${ }^{1,3}$

\subsection{Roof WFS approximation}

The theoretical model of the P-WFS becomes simpler when instead of the four-sided pyramidal prism one assumes two orthogonally placed two-sided roof prisms. ${ }^{4-6}$ Thanks to the physical decoupling of the prisms and their orthogonal placement with respect to each other, the two signal sets $S_{x}$ and $S_{y}$ are independent and contain information about the phase $\phi$ only in $x$ - and only in $y$-direction correspondingly. Due to the symmetry, we consider $S_{x}$ measurements only.

Theorem 1. Under the roof sensor assumption, the P-WFS data $S_{x}^{n, l, c}$ is approximated as

$$
S_{x}^{\{n, l, c\}}(x, y)=\int_{-B(y)}^{+B(y)} \frac{\sin \left[\phi\left(x^{\prime}, y\right)-\phi(x, y)\right] k_{\{n, l, c\}}\left(x^{\prime}-x\right)}{\pi\left(x-x^{\prime}\right)} d x^{\prime}
$$

where the functions $k_{\{n, l, c\}}$ are defined as $k_{n}(x)=1, k_{l}(x)=\operatorname{sinc}\left(\alpha_{\lambda}(x)\right), k_{c}(x)=J_{0}\left(\alpha_{\lambda}(x)\right)$. Here the superscripts $\{n, l, c\}$ denote the cases of no modulation applied, a linear and a circular modulation of amplitude $\alpha=\frac{b \lambda}{D}$ with a positive integer $b ;\{-B(y),+B(y)\}$ denote the boundaries of the pupil images for a fixed $y$, $\alpha_{\lambda}=\frac{2 \pi \alpha}{\lambda}$, and $J_{0}$ denotes the zero-order Bessel function of the first kind.

Proof. See. ${ }^{1,4,5}$

\subsection{Closed loop approximation}

An additional assumption of small wavefront distortions $\phi \ll 1$, as expected in the closed loop, allows to linearize the models of the pyramid sensor measurements.

Theorem 2. Under the roof sensor and closed loop assumptions, the linearized pyramid sensor data is given as

$$
S_{x}(x, y)=\frac{1}{\pi} \int_{-B(y)}^{B(y)} \frac{\left[\phi\left(x^{\prime}, y\right)-\phi(x, y)\right] k_{\{n, l, c\}}\left(x^{\prime}-x\right)}{x-x^{\prime}} d x^{\prime} .
$$

Proof. See. ${ }^{1,4,5}$ 


\subsection{Infinite telescope approximation}

Assuming an infinite telescope size $B(y) \rightarrow \infty$, one can simplify the forward models further.

Theorem 3. Under the roof sensor, closed loop, and infinite telescope size assumptions, the P-WFS data is given as

$$
S_{x}^{\{n, l, c\}}(x, y)=\left(\phi(\cdot, y) * \frac{k_{\{n, l, c\}}(\cdot)}{\pi \cdot}\right)(x, y),
$$

where $*$ denotes the convolution operator.

Proof. See. ${ }^{1,4,5}$

\subsection{Subaperture discretization}

So far we have considered the continuous model of the data and neglected the finite sampling of the sensor. What we measure in practice is the averaged data values over the subapertures. Following the approach in, ${ }^{4}$ we consider the sensor data $S_{x}^{\{n, l, c\}}$ as discrete functions evaluated in the (discrete) middle points $\{\bar{x}, \bar{y}\}$ of WFS subapertures.

We obtain the discrete sensor data $\bar{S}_{x}^{\{n, l, c\}}$ at the discrete space variable $\bar{x}$ in the following two steps. In the first step, we average the continuous data $S_{x}^{\{n, l, c\}}$ over the subapertures, which is mathematically represented by a convolution of $S_{x}^{\{n, l, c\}}$ with a characteristic function $\Pi$ of the interval $[-1 / 2,1 / 2]$, namely

$$
\begin{aligned}
\bar{S}_{x}^{\{n, l, c\}}(x) & =\left(S_{x}^{\{n, l, c\}}(\cdot) * \frac{1}{d} \Pi\left(\frac{\dot{y}}{d}\right)\right)(x) \\
& =\frac{1}{d} \int_{-\infty}^{+\infty} S_{x}^{\{n, l, c\}}\left(x^{\prime}\right) \Pi\left(\frac{x-x^{\prime}}{d}\right) d x^{\prime} \\
& =\frac{1}{d} \int_{x-d / 2}^{x+d / 2} S_{x}^{\{n, l, c\}}\left(x^{\prime}\right) d x^{\prime} .
\end{aligned}
$$

In the second step, from the averaged data values $\bar{S}_{x}^{\{n, l, c\}}(x)$ given at continuous space variable $x$ we pick a set of discrete values $\left\{\hat{S}_{x}^{\{n, l, c\}}\right\}=\left\{\bar{S}_{x}^{\{n, l, c\}}(\bar{x})\right\}$ in the middle points $\bar{x}$ of the subapertures. Mathematically, this step is represented by a multiplication of the averaged data $\bar{S}_{x}^{\{n, l, c\}}(x)$ with the so called sampling function $T_{d}$,

$$
\hat{S}_{x}^{\{n, l, c\}}(x)=\bar{S}_{x}^{\{n, l, c\}}(x) \cdot T_{d}(x) .
$$

The sampling function $T_{d}$, also known as the Dirac comb, from the mathematical point of view is a distribution, or a generalized function, and is defined as an infinite sum of the shifted delta distributions,

$$
T_{d}(x):=\sum_{k=-\infty}^{+\infty} \delta(x-k d)=\frac{1}{d} T\left(\frac{x}{d}\right) .
$$

Therefore, by a multiplication of the averaged data $\bar{S}_{x}^{\{n, l, c\}}(x)$ with $T_{d}(x)$, we pick a discrete set of values of $\bar{S}_{x}^{\{n, l, c\}}(\bar{x})$ in a discrete set of points $\{\bar{x}\}=\left\{x \mid \frac{x}{d} \in \mathbb{Z}\right\}$ representing the middle points of the sensor subapertures.

\section{FOURIER DOMAIN REPRESENTATION}

Now we can perform the Fourier transform on the (discretized) sensor data $\hat{S}_{x}^{\{n, l, c\}}(x)$. Note that due to the finite sampling (i.e., subaperture discretization) of the WFS, the spectrum of the measured sensor data contains only certain (discrete) frequencies $\bar{u}$ sampled in the interval $\left[-u_{\text {cut }}, u_{\text {cut }}\right]$ with a sampling size $u_{\text {step }}:=1 / D$, determined by the telescope diameter $D$. The cut-off frequency $u_{c u t}$ is determined by the sensor subaperture size $d$ as $u_{\text {cut }}=1 /(2 d)$. In the modulated case, let the parameter $u_{\text {mod }}>0$ be defined as $u_{\text {mod }}=\alpha / \lambda=b / D$, where $b$ is a positive integer. The parameter $u_{\text {mod }}$ defines the frequency at which the transition between the two regimes (slope versus phase mode) of the pyramid-type sensor happens. 


\subsection{Spectrum of continuous data}

Theorem 4. For each of the modulation scenarios, the spectrum of the continuous sensor data is given as a product of the wavefront spectrum with a corresponding filter function $g_{x}^{n, l, c}$,

$$
\left(\mathcal{F} S_{x}^{\{n, l, c\}}\right)(u)=(\mathcal{F} \phi)(u) \cdot g_{\{n, l, c\}}(u),
$$

where the Fourier domain filters $g_{\{n, l, c\}}$, corresponding to the sensor without modulation, with linear and circular modulation of radius $\alpha$ respectively, are given as

$$
\begin{gathered}
g_{n}(u)=i \operatorname{sgn}(u), \forall u \in\left[-u_{\text {cut }}, u_{\text {cut }}\right], \\
g_{l}(u)= \begin{cases}i \operatorname{sgn}(u), & |u|>u_{\text {mod }}, \\
i u / u_{\text {mod }}, & |u| \leq u_{\text {mod }},\end{cases} \\
g_{c}(u)= \begin{cases}i \operatorname{sgn}(u), & |u|>u_{\text {mod }}, \\
\frac{2 i}{\pi} \arcsin \left(u / u_{\text {mod }}\right), & |u| \leq u_{\text {mod }} .\end{cases}
\end{gathered}
$$

Proof. The proof consists in applying the Fourier convolution theorem to Eq. (3). For details we refer to. ${ }^{1,4}$

\subsection{Spectrum of averaged continuous data}

Theorem 5. For each of the modulation scenarios, the spectrum of the averaged continuous sensor data $S_{x}^{\{n, l, c\}}$ is given as the point-wise product

$$
\left(\mathcal{F} \bar{S}_{x}^{\{n, l, c\}}\right)(u)=(\mathcal{F} \phi)(u) \cdot h_{\{n, l, c\}}(u),
$$

of the wavefront spectrum, evaluated at a discrete set of frequencies $u$, with the corresponding discrete filter function $h_{n, l, c}$ given as

$$
h_{\{n, l, c\}}(\bar{u})=g_{\{n, l, c\}}(u) \cdot \operatorname{sinc}(d u) .
$$

Proof. The spectrum of the averaged continuous sensor data $\bar{S}_{x}^{\{n, l, c\}}$ is obtained from (4) as

$$
\begin{aligned}
\left(\mathcal{F} \bar{S}_{x}^{\{n, l, c\}}\right)(u) & =\left(\mathcal{F}\left(S_{x}^{\{n, l, c\}}(\cdot) * \frac{1}{d} \Pi\left(\frac{\dot{d}}{d}\right)\right)(x)\right)(u) \\
& =\left(\mathcal{F} S_{x}^{\{n, l, c\}}\right)(u) \cdot\left(\mathcal{F} \frac{1}{d} \Pi(\dot{\bar{d}})\right)(u) \\
& =\left(\mathcal{F} S_{x}^{\{n, l, c\}}\right)(u) \cdot \operatorname{sinc}(d u),
\end{aligned}
$$

Using (7), we obtain the statement of the Theorem.

\subsection{Spectrum of discrete data}

Theorem 6. For any modulation scenario, the spectrum of the discretized pyramid sensor data $\hat{S}_{x}^{\{n, l, c\}}$ is a convolution of the spectrum of the averaged continuous sensor data $\bar{S}_{x}^{\{n, l, c\}}$ with the sampling function

$$
\left(\mathcal{F} \hat{S}_{x}^{\{n, l, c\}}\right)(u)=\left(\mathcal{F} \bar{S}_{x}^{\{n, l, c\}}(\cdot) * T(d \cdot)\right)(u)
$$


Proof. Applying the Fourier convolution theorem to (5) and using the fact that the Fourier transform of the sampling function $T_{d}$ is again a sampling function,

$$
\left(\mathcal{F} T_{d}(\cdot)\right)(u)=\frac{1}{d} T_{\frac{1}{d}}(u)=T(d u) .
$$

We obtain

$$
\begin{aligned}
\left(\mathcal{F} \hat{S}_{x}^{\{n, l, c\}}\right)(u) & =\left(\mathcal{F}\left[\bar{S}_{x}^{\{n, l, c\}}(\cdot) \cdot T_{d}(\cdot)\right]\right)(u) \\
& =\left(\left(\mathcal{F} \bar{S}_{x}^{\{n, l, c\}}\right)(\cdot) *\left(\mathcal{F} T_{d}\right)(\cdot)\right)(u) \\
& =\left(\left(\mathcal{F} \bar{S}_{x}^{\{n, l, c\}}\right)(\cdot) * T(d \cdot)\right)(u) .
\end{aligned}
$$

Note that the convolution of $\mathcal{F} \bar{S}_{x}^{\{n, l, c\}}$ with the sampling function $T(d u)$ simply denotes that discrete values of $\mathcal{F} \bar{S}_{x}^{\{n, l, c\}}$ corresponding to subaperture centers are assigned to the discrete spectrum $\left(\mathcal{F} \hat{S}_{x}^{\{n, l, c\}}\right)$.

The Fourier domain filters $h_{\{n, l, c\}}$ for different modulation scenarios with modulations 0 and $12 \lambda / D$ are illustrated in Figure 1.

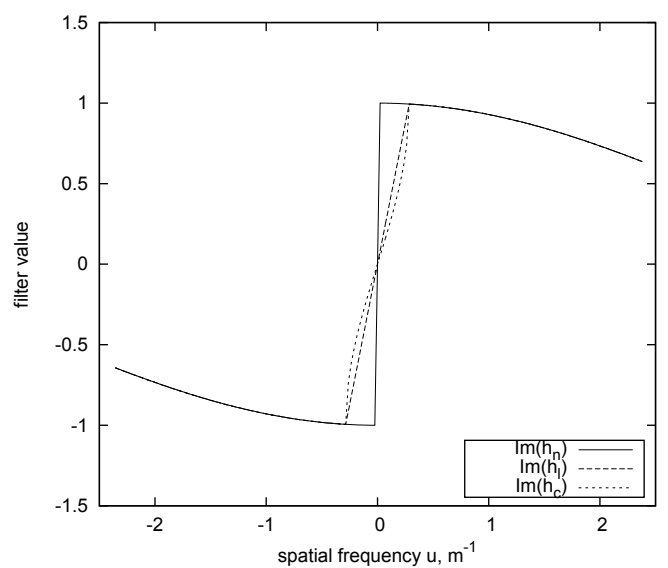

(a)

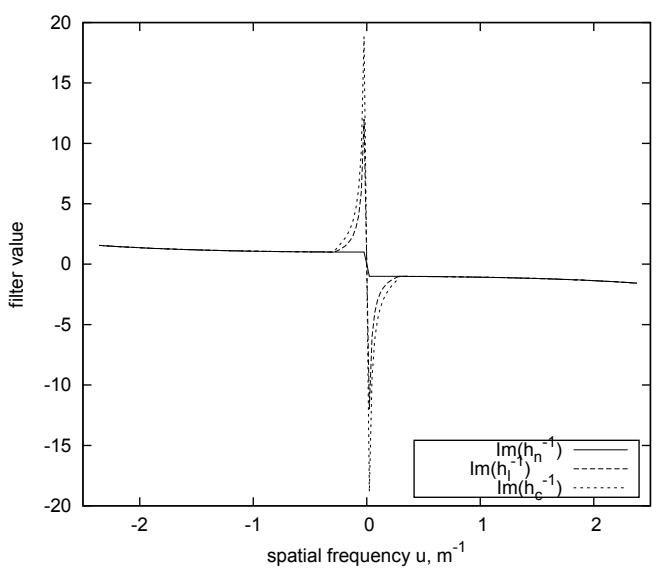

(b)

Figure 1: (a) Fourier domain filters $h_{n, l, c}$. (b) Inverse Fourier domain filters $h_{n, l, c}^{-1}$ for modulation $12 \lambda / D$.

\section{TWO NOVEL ALGORITHMS}

Our PFTR/CLIF method is based on the Fourier domain relation between the spectra of the discrete sensor data and the wavefront described in the previous section. The reconstruction consists essentially in the application of the corresponding inverse Fourier domain filter. This inversion procedure can be performed in two different ways - either as a multiplication on the Fourier domain, or as a convolution on the spatial domain. The two presented algorithms correspond to these two different implementations of the same reconstruction idea. Though mathematically the algorithms are equivalent, they have different computational complexities, which is why it makes sense to distinguish them.

\subsection{Pyramid Fourier Transform Reconstructor}

Theorem 7. For any modulation scenario, the wavefront can be reconstructed from the discrete sensor data $\hat{S}_{x}^{\{n, l, c\}}$ as

$$
\phi_{x}^{r e c}(x)=\left(\mathcal{F}^{-1}\left[\left(\mathcal{F} \hat{S}_{x}^{\{n, l, c\}}\right)(u) \cdot h_{\{n, l, c\}}^{-1}(u)\right]\right)(x),
$$


where the inverse Fourier domain filters $h_{\{n, l, c\}}^{-1}$, corresponding to the sensor without, with linear and circular modulation of radius a respectively, are defined on $\forall u \in\left[-u_{\text {cut }}, 0\right) \cup\left(0, u_{\text {cut }}\right]$ as

$$
h_{\{n, l, c\}}^{-1}=g_{\{n, l, c\}}^{-1}(\bar{u}) \cdot \operatorname{sinc}^{-1}(d \bar{u})
$$

with the filter functions $g_{\{n, l, c\}}^{-1}$ given by

$$
\begin{gathered}
g_{n}^{-1}(u)=-i \operatorname{sgn}(u), \\
g_{l}^{-1}(u)= \begin{cases}-i \operatorname{sgn}(u), & |u|>u_{\text {mod }}, \\
-i u_{\text {mod }} / u, & |u| \leq u_{\text {mod }},\end{cases} \\
g_{c}^{-1}(u)= \begin{cases}-i \operatorname{sgn}(u), & |u|>u_{\text {mod }}, \\
-\frac{i \pi}{2 \arcsin \left(u / u_{\text {mod }}\right)}, & |u| \leq u_{\text {mod }}\end{cases}
\end{gathered}
$$

and containing singularities at $u=0$,

$$
\begin{array}{cc}
\lim _{u \rightarrow 0^{-}} g_{n}^{-1}(u)=-i, & \lim _{u \rightarrow 0^{+}} g_{n}^{-1}(u)=i, \\
\lim _{u \rightarrow 0^{-}} g_{l}^{-1}(u)=\infty, & \lim _{u \rightarrow 0^{+}} g_{l}^{-1}(u)=-\infty, \\
\lim _{u \rightarrow 0^{-}} g_{c}^{-1}(u)=\infty, & \lim _{u \rightarrow 0^{+}} g_{c}^{-1}(u)=-\infty .
\end{array}
$$

Proof. Using the results in Theorems 5 and 6, and multiplying (11) from both sides with the corresponding discretized inverse Fourier domain filter $h_{\{n, l, c\}}^{-1}$, we obtain the wavefront spectrum in the form

$$
(\mathcal{F} \phi)(u)=\left(\mathcal{F} \bar{S}_{x}^{\{n, l, c\}}\right)(u) \cdot h_{\{n, l, c\}}^{-1}(u)
$$

Then, applying the inverse Fourier transform, we obtain the reconstruction formula

$$
\phi_{x}^{r e c}(x)=\left(\mathcal{F}^{-1}(\mathcal{F} \phi)\right)(x) .
$$

The expressions for the filter functions $g_{\{n, l, c\}}^{-1}$ and $h_{\{n, l, c\}}^{-1}$ are obtained from Theorems 5 and 6 .

Figure 1 illustrates the inverse filters $h_{\{n, l, c\}}^{-1}$ for different modulation scenarios with modulations 0 and $12 \lambda / D$.

Based on Theorem 7, the PFTR algorithm consists in the following five steps:

\section{Algorithm 1: PFTR.}

1. Computation of the $1 \mathrm{D}$ inverse Fourier domain filter $h_{\{n, l, c\}}^{-1}(u)$ according to (15). This step has to be performed only once for the chosen AO system and can be pre-computed before the AO loop starts.

2. Computation of the spectrum $\left\{\left(\mathcal{F} S_{x}\right)(u),\left(\mathcal{F} S_{y}\right)(u)\right\}$ of the sensor data. The 1D Fourier transform (FT) is applied row-wise to the sensor data $S_{x}$ and column-wise to data $S_{y}$.

3. Multiplication of the sensor data spectrum $\left\{\left(\mathcal{F} S_{x}\right)(u),\left(\mathcal{F} S_{y}\right)(u)\right\}$ with the inverse Fourier domain filter $h_{\{n, l, c\}}^{-1}(u)$. The multiplication is performed row-wise for the spectrum of data $S_{x}$ and column-wise for the spectrum of data $S_{y}$.

4. Computation of the Inverse Fourier Transform (IFT) of the result.

5. Averaging of the two wavefronts reconstructed from $S_{x}$ and $S_{y}$ sensor data. 


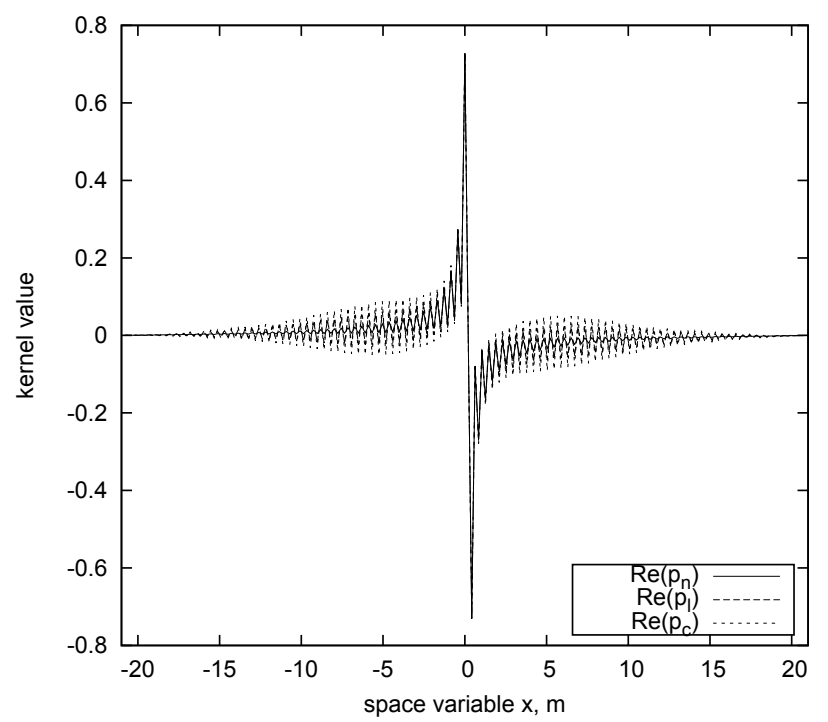

Figure 2: Numerically evaluated space domain kernels $p_{\{n, l, c\}}$ for modulation $4 \lambda / D$.

The difference of our PFTR algorithm compared to the FTR algorithm presented in $^{7}$ is in the filter functions used. In $^{7}$ the authors assumed the pyramid sensor with a large amount of modulation applied. Since in this regime the pyramid sensor acts similar to the Shack-Hartmann sensor, i.e., measures the slopes of the wavefront, the authors in $^{7}$ simply used the Fourier domain filter derived for the Shack-Hartmann sensor. In our approach we use the filter functions derived from the forward mathematical models of the pyramid sensor. Moreover, our approach is general, since it is suited for any modulation scenario and any amount of modulation applied.

\subsection{Convolution with the Linearized Inverse Filter}

Theorem 8. For any modulation scenario, the wavefront can be reconstructed from the discrete sensor data $\bar{S}_{x}^{\{n, l, c\}}$ as

$$
\phi_{x}^{r e c}(\bar{x})=\left(\bar{S}_{x}^{\{n, l, c\}}(\cdot, y) * p_{\{n, l, c\}}(\cdot)\right)(\bar{x}),
$$

where the convolution kernel $p$ is defined as

$$
\begin{aligned}
p_{\{n, l, c\}}(x) & :=\left(\mathcal{F}^{-1} h_{\{n, l, c\}}^{-1}\right)(\bar{x}) \\
& =\left[\mathcal{F}^{-1}\left(g^{-1} \cdot \operatorname{sinc}^{-1}\right)\right](x) \\
& =\left[\left(\mathcal{F}^{-1} g^{-1}\right)(\cdot) *\left(\mathcal{F}^{-1} \operatorname{sinc}^{-1}\right)(\cdot)\right](x) .
\end{aligned}
$$

Proof. The reconstruction formula (14) can be transformed to the spatial domain using the Fourier convolution theorem as follows

$$
\begin{aligned}
\phi_{x}^{r e c}(x, y) & =\left(\mathcal{F}^{-1}(\mathcal{F} \phi)\right)(x, y) \\
& =\left(\mathcal{F}^{-1}\left[\left(\mathcal{F} \bar{S}_{x}^{\{n, l, c\}}\right)(\bar{u}, y) \cdot h_{\{n, l, c\}}^{-1}(\bar{u})\right]\right)(x, y) \\
& =\left(\left[\mathcal{F}^{-1}\left(\mathcal{F} S_{x}^{\{n, l, c\}}\right)\right](\cdot, y) *\left[\mathcal{F}^{-1} h_{\{n, l, c\}}^{-1}\right](\cdot, y)\right)(x, y) \\
& =\left(S_{x}^{\{n, l, c\}}(\cdot, y) *\left(\mathcal{F}^{-1} h_{\{n, l, c\}}^{-1}\right)(\cdot)\right)(x, y),
\end{aligned}
$$

which finishes the proof. 
Figure 2 illustrates the spatial domain kernels $p_{\{n, l, c\}}$ for different modulation scenario with modulation $4 \lambda / D$.

Based on Theorem 8, the CLIF algorithm consists of the following three steps:

Algorithm 2: CLIF.

1. Computation of the 1D discrete spatial domain kernel $p_{\{n, l, c\}}(\bar{x})$ according to (22). This step has to be performed only once for the chosen $\mathrm{AO}$ system and can be pre-computed before the AO loop starts.

2. Computation of two wavefronts $\left\{\phi_{x}^{r e c}, \phi_{y}^{r e c}\right\}$ from two data sets $\left\{S_{x}, S_{y}\right\}$ according to (21). The convolution is performed row-wise for data $S_{x}$ and column-wise for data $S_{y}$.

3. Averaging of the two reconstructed wavefronts.

\section{SIMULATED CLOSED LOOP PERFORMANCE}

To test the quality of our reconstruction method in a closed loop setting, we use the end-to-end simulator OCTOPUS developed by the European Southern Observatory (ESO). ${ }^{8}$ The used simulation parameters are summarized in Table 1.

We consider an XAO system with telescope diameter of $D=42 \mathrm{~m}$ and a pyramid sensor. The software simulates a 9-layer atmospheric model, each layer being a random realization of the von Karman power spectrum. We test the closed loop performance of our reconstruction method for a $200 \times 200$ pyramid sensor with the modulation radius $\alpha=4 \lambda / D$. The XAO system runs with the frame rate of $3 \mathrm{kHz}$. DM influence functions $I F$ of the form

$$
\begin{aligned}
I F(x, y) & =\left[1-|x|^{3.805}+3.74 \ln |x| \cdot|x|^{2.451}\right] \\
& \times\left[1-|y|^{3.805}+3.74 \ln |y| \cdot|y|^{2.451}\right]
\end{aligned}
$$

are assumed. Note that the form of the DM influence functions was predefined in our tests by the reference results and reference parameters provided by ESO.

We evaluate the quality in a wide range of atmospheric conditions and photon flux levels as given in Table 1. For the temporal control of the closed loop we use a simple integrator, the gain is optimized manually (on 100 time steps) with a resolution of 0.1 . The results are obtained simulating 500 time steps. The quality metric used is the Long-Exposure (LE) Strehl ratio, which is computed as the average on-axis Strehl ratio for all the performed time steps.

Note that the less the photon flux is, the higher is the impact of the shot noise on the sensor data. It is clear that the reconstruction from the data with more noise is less trustable than from the noiseless data. Therefore, in the low flux case it makes sense to apply a smaller control gain. This allows to regularize (smoothen) the DM shape. Hence, gain optimization has to be performed for each photon flux level (shot noise level, respectively) separately.

We compare the quality of our CLIF/PFTR method with that of the P-CuReD algorithm, which were reported in, ${ }^{3}$ and the MVM method, which were provided to us by ESO. Table 2 gives a comparison of the LE Strehl ratios obtained with three methods for three 'ESO-standard atmospheres' and varying photon flux. The result obtained for the median atmosphere is visualized in Figure 3. It shows that, compared to both MVM and P-CuReD, our CLIF/PFTR method achieves the slightly lower quality in the high photon flux case. However, in case of low photon flux the quality of CLIF/PFTR is slightly lower than that of P-CuReD, but much higher compared to MVM.

Note that the MVM results we present in the paper were provided to us by the ESO, as a benchmark for new reconstruction methods. The MVM method which was used is modal reconstructor, using maximum a posteriori (MAP) priors (von Karman covariance matrix), with a hyper-parameter tuning to account for the fact that the atmospheric statistic are for open loop operation, whereas the system is used in closed loop. An interaction 
Table 1: Simulation parameters.

\begin{tabular}{ll}
\hline telescope diameter D & $42 \mathrm{~m}$ \\
central obstruction & $28 \%$ \\
science target & on-axis (SCAO) \\
science band & $\mathrm{K}, \lambda_{\text {Strehl }}=2200 \mathrm{~nm}$ \\
sensing band & $\mathrm{R}, \lambda_{W F S}=700 \mathrm{~nm}$ \\
WFS & pyramid \\
type of modulation & circular \\
controller & integrator \\
atmospheric model & von Karman \\
number of simulated layers & 9 \\
outer scale $L_{0}$ & $25 \mathrm{~m}$ \\
Fried radius $r_{0}$ at $\lambda=500 \mathrm{~nm}$ & \\
$\quad$ for good atmosphere & $0.172 \mathrm{~m}$ \\
$\quad$ for median atmosphere & $0.129 \mathrm{~m}$ \\
$\quad$ for bad atmosphere & $0.094 \mathrm{~m}$ \\
number of subapertures $n_{s}$ & $200 \times 200=40000$ \\
number of active subapertures $n$ & 28800 \\
number of DM actuators $n_{a}$ & 29618 \\
frame rate & $3 \mathrm{kHz}$ \\
DM delay & 1 \\
photon flux & {$[5,10,50,100,1000,10000]$} \\
read noise & $2.8 \mathrm{e}-/$ pixel \\
modulation radius in $\lambda / D$ & 4 \\
atmosphere & {$[$ good, median, bad] } \\
iterations per simulation & 500 \\
\hline
\end{tabular}

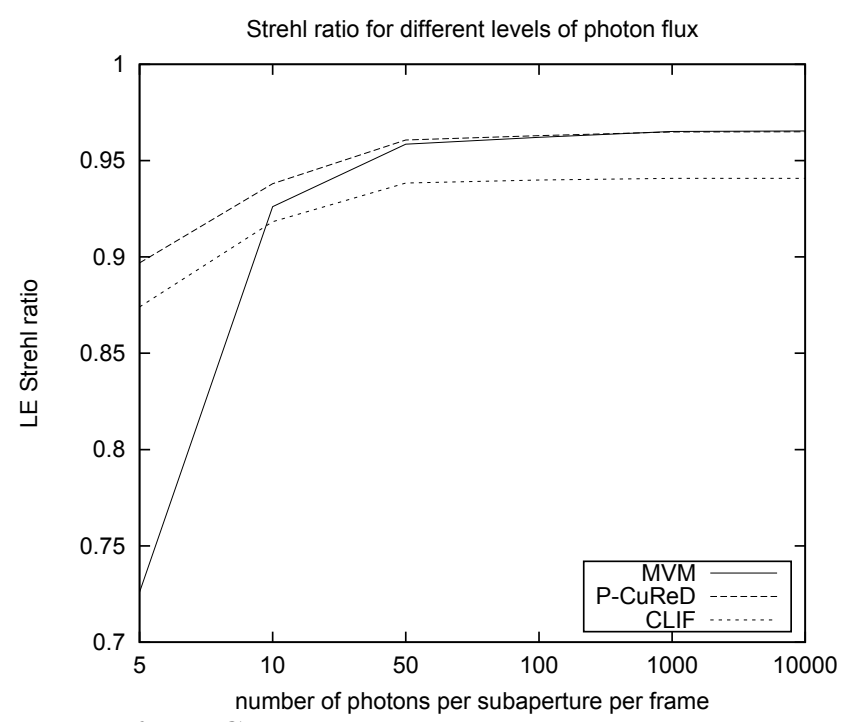

Figure 3: Close loop performance of the CLIF algorithm in comparison with that of the MVM and P-CuReD methods for the case of median atmospheric conditions and various photon fluxes.

matrix connects KarhunenLoève (KL) modes (as best approximated by the DM) to the measured sensor response. With the regularized inverse of this matrix (control matrix) one reconstructs the actuator commands from the measured sensor data. A single gain value was used for all KL modes. A single matrix was used for all fluxes in the MVM case, just optimizing a single loop gain. 
Table 2: Comparison of the LE Strehl ratios obtained with the MVM, the P-CuReD, and the CLIF method for different atmospheres and photon fluxes using the indicated simulation parameters.

\begin{tabular}{|c|c|c|c|c|c|c|c|c|c|}
\hline Atmosphere & \multicolumn{3}{|c|}{ good } & \multicolumn{3}{|c|}{ median } & \multicolumn{3}{|c|}{ bad } \\
\hline Photon flux & MVM & P-CuReD & CLIF & MVM & P-CuReD & CLIF & MVM & P-CuReD & CLIF \\
\hline 5 & 0.8709 & 0.9317 & 0.9209 & 0.7260 & 0.8969 & 0.8740 & 0.4355 & 0.8203 & 0.7766 \\
\hline 10 & 0.9524 & 0.9582 & 0.9452 & 0.9261 & 0.9380 & 0.9183 & 0.8785 & 0.9006 & 0.8628 \\
\hline 50 & 0.9742 & 0.9748 & 0.9616 & 0.9585 & 0.9607 & 0.9384 & 0.9260 & 0.9344 & 0.9021 \\
\hline 100 & 0.9765 & 0.9766 & 0.9632 & 0.9621 & 0.9630 & 0.9399 & 0.9335 & 0.9378 & 0.9048 \\
\hline 1000 & 0.9785 & 0.9780 & 0.9640 & 0.9651 & 0.9649 & 0.9408 & 0.9384 & 0.9405 & 0.9070 \\
\hline 10000 & 0.9788 & 0.9781 & 0.9640 & 0.9654 & 0.9650 & 0.9408 & 0.9387 & 0.9408 & 0.9071 \\
\hline
\end{tabular}

\section{COMPUTATIONAL COMPLEXITY}

In this Section we analyse and compare the properties of the CLIF/PFTR methods with the emphasis on their computational complexities, and compare them to other existing algorithms, especially MVM, as listed in Tables 3 and 4.

The CLIF method consists in a row-wise convolution of the sensor data $S_{x}$ and a column-wise convolution of the sensor data $S_{y}$ with a 1D kernel $p$. The obtained results are then averaged. The full kernel $p$ consists of $\sqrt{n}$ points, where $n$ denotes the number of wavefront sensor active subapertures. Hence, the CLIF method requires $4 n \sqrt{n}$ operations for the convolution, and $n$ operations for the averaging, which makes together $4 n \sqrt{n}+n$ operations to be performed. Therefore, the complexity of the CLIF method scales as $O\left(n^{3 / 2}\right)$ with the number $n$ of active subapertures of the wavefront sensor, which is significantly more efficient compared to the MVM method.

Moreover, as one can see from Figure 2, a lot of the kernel values are rather small, and therefore could be neglected in the convolution. In this case a smaller number of operations would have to be performed. Our tests in the XAO setting have showed that the method reaches the same correction quality if only $60 \%$ of the largest kernel values are used. Note that for other settings this number could be different.

In the PFTR method the reconstruction is performed on the Fourier domain, and the computational complexity is further reduced. First, one has to compute 1D Fourier transforms of the sensor data, which requires $O(n \log n)$ operations in case the FFT algorithm is used. Then, multiplication of the sensor data spectra $\left(\mathcal{F} S_{x, y}\right.$ with the inverse filter $h_{n, l, c}^{-1}$ requires $n$ operations. Further, computation of the inverse Fourier transform of the filtered data spectra requires again $O(n \log n)$ operations. Finally, averaging of two reconstructions costs $n$ operations. Therefore, the total complexity of the Fourier domain reconstructor scales as $O(n \log n)$ with the number of $n$ of active subapertures of the wavefront sensor.

Thus, in comparison with the P-CuReD with the complexity $O(n)$, and the MVM method with the complexity $O\left(n^{2}\right)$, the CLIF/PFTR method takes up an intermediate position. In Table 3 we compare the number of operations per frame required by various algorithms in the considered XAO setting. Here one can clearly see the drastic reduction of computational load which the algorithms based on the mathematical forward model of the sensor can achieve compared to the hardware-based MVM approach.

Table 3: Comparison of the number of operations per frame required required by different reconstruction algorithms in the specific XAO setting considered in this paper.

\begin{tabular}{lccc}
\hline Algorithm & \multicolumn{3}{c}{$\begin{array}{c}\text { Number of operations } \\
\text { in XAO setting }\end{array}$} \\
\hline MVM & $4 n_{a} n^{*}$ & $3.4120 \mathrm{e}+09$ & $100 \%$ \\
P-CuReD & $(4 c-2) n+20 n^{*}$ & 1324800 & $0.0388 \%$ \\
CLIF & $4 n \sqrt{n}+n$ & $1.9579 \mathrm{e}+07$ & $0.5738 \%$ \\
\hline${ }^{*} n_{a}=29618, n=28800, c=7$
\end{tabular}

Additionally, in Table 4 we compare the computational complexities, applicability and achievable quality of our two novel algorithms to other existing methods. 
Table 4: Comparison of the currently existing algorithms for wavefront reconstruction from pyramid sensor data in terms of their flexibility, computational complexity, need in time consuming fine tuning when atmospheric conditions change, and the achieved closed loop performance.

\begin{tabular}{|c|c|c|c|c|c|}
\hline Algorithm & \multicolumn{3}{|c|}{ Modulation } & Complexity & $\begin{array}{l}\text { Quality in end-to-end } \\
\text { simulations in } \\
\text { the XAO ELT setting* }\end{array}$ \\
\hline Matrix-Vector Multiplication (MVM) & + & + & + & $O\left(n^{2}\right)$ & baseline \\
\hline Fourier Transform Reconstructor (FTR) & - & - & + & $O(n \log n)$ & ?, slightly lower for $\mathrm{VLT}^{*}$ \\
\hline Preprocessed CuReD (P-CuReD) ${ }^{1,3}$ & + & + & + & $O(n)$ & same / higher in low flux \\
\hline Conv. with Lin. Inverse Filter $(\mathrm{CLIF})^{1,9}$ & + & + & + & $O\left(n^{3 / 2}\right)$ & slightly lower / higher in low flux \\
\hline Pyramid FTR $(\text { PFTR })^{9}$ & + & + & + & $O(n \log n)$ & same as CLIF \\
\hline Conj. Gradient for Normal Eq. (CGNE) $)^{1,10,11}$ & $(+)$ & + & + & $O\left(n^{3 / 2}\right)$ & work in progress \\
\hline Hilbert Transform Rec. (HTR) & + & - & - & $O(n \log n)$ & slightly lower \\
\hline Finite Hilbert Transform Rec. $(\text { FHTR })^{1}$ & + & - & - & $O\left(n^{3 / 2}\right)$ & work in progress \\
\hline Singular Value Type Rec. $(\mathrm{SVTR})^{12}$ & + & - & - & $O\left(n^{3 / 2}\right)$ & same as CLIF \\
\hline Pyramid Kaczmarz Iteration $(\mathrm{PKI})^{10,11}$ & + & + & + & $O\left(n^{3 / 2}\right)$ & same as / better than P-CuReD \\
\hline
\end{tabular}

* XAO ELT setting: $D=42 \mathrm{~m}, n_{\text {sub }}=200 \times 200, d=0.21 \mathrm{~m}$

** VLT setting: $D=8 \mathrm{~m}, n_{\text {sub }}=40 \times 40, d=0.5 \mathrm{~m}$

To summarize, the CLIF/PFTR method is general, parameter-free, highly parallelizable and significantly faster than MVM. These features make our method especially attractive for usage in the real-time extreme adaptive optics.

\section{CONCLUSIONS}

The new methods we introduced in this paper, CLIF and PFTR, can reconstruct the wavefront for both the modulated and non-modulated pyramid-type sensor data.

The computational complexity of the method depends on the chosen implementation and the properties of the spatial domain kernel, and can be scaled from $O\left(n^{3 / 2}\right)$ down to $O(n \log (n))$, where $n$ denotes the number of active subapertures of the wavefront sensor. Therefore, with respect to the number of operations to be performed, the CLIF/PFTR method occupies an intermediate position between the MVM $\left(O\left(n^{2}\right)\right)$ and the P-CuReD $(O(n))$ algorithms. Compared to the MVM method with the complexity of $O\left(n^{2}\right)$, our approach is significantly more efficient.

From the closed loop quality tests, performed for various atmospheric conditions and photon fluxes, we have seen that the quality of the CLIF method is slightly lower that the quality of the MVM and the P-CuReD algorithms in the high flux case, and is much better than the quality of MVM in the low flux regime. We would like to point out here, that the difference in the quality obtained with the CLIF/PFTR method compared to the $\mathrm{P}-\mathrm{CuReD}$ is rather small, therefore it is not clear if this feature will stay the same when the algorithms are tested with real data on sky.

Analysis of the noise propagation properties of the algorithms and development of RTC prototypes for the speed comparison to MVM are the points of our further research.

\section{REFERENCES}

[1] Shatokhina, I., Fast Wavefront Reconstruction Algorithms for eXtreme Adaptive Optics, PhD thesis (Nov 2014).

[2] Korkiakoski, V., Vérinaud, C., Le Louarn, M., and Conan, R., "Comparison between a model-based and a conventional pyramid sensor reconstructor," Applied Optics 46(24), 6176-6184 (2007).

[3] Shatokhina, I., Obereder, A., Rosensteiner, M., and Ramlau, R., "Preprocessed cumulative reconstructor with domain decomposition: a fast wavefront reconstruction method for pyramid wavefront sensor," Appl. Opt. 52, 2640-2652 (Apr 2013).

[4] Vérinaud, C., "On the nature of the measurements provided by a pyramid wave-front sensor," Optics Communications 233, 27-38 (2004). 
[5] Burvall, A., Daly, E., Chamot, S. R., and Dainty, C., "Linearity of the pyramid wavefront sensor," Optics Express 14 (25), 11925-11934 (2006).

[6] Phillion, D. W. and Baker, K., "Two-sided pyramid wavefront sensor in the direct phase mode," in [Proc. SPIE 6272, Advances in Adaptive Optics II], 627228 (2006).

[7] Quirós-Pacheco, F., Correia, C., and Esposito, S., "Fourier transform-wavefront reconstruction for the pyramid wavefront sensor," in [Proceedings of the First AO4ELT Conference], 07005 (2010).

[8] Le Louarn, M., Vérinaud, C., Korkiakoski, V., Hubin, N., and Marchetti, E., "Adaptive optics simulations for the European Extremely Large Telescope," in [Proc. SPIE 6272, Advances in Adaptive Optics II], 627234 (2006).

[9] Shatokhina, I. and Ramlau, R., "Convolution- and fourier-transform-based reconstructors for pyramid wavefront sensor," Appl. Opt. 56, 6381-6390 (Aug 2017).

[10] Hutterer, V., Shatokhina, I., and Ramlau, R., "Real-time adaptive optics with pyramid wavefront sensor: Theoretical analysis of pyramid forward model," in preparation.

[11] Hutterer, V., Shatokhina, I., and Ramlau, R., "Real-time adaptive optics with pyramid wavefront sensor: Accurate wavefront reconstruction with iterative methods," in preparation.

[12] Hutterer, V. and Ramlau, R., "Wavefront reconstruction from non-modulated pyramid wavefront sensor data using a singular value type expansion," submitted. 